\title{
Erratum to: Glycyrrhizin, inhibitor of high mobility group box-1, attenuates monocrotaline-induced pulmonary hypertension and vascular remodeling in rats
}

Pil-Sung Yang ${ }^{1}$, Dae-Hoon Kim¹, Yong Joon Lee ${ }^{2}$, Sang-Eun Lee ${ }^{1}$, Won Jun Kang ${ }^{3}$, Hyuk-Jae Chang ${ }^{1,4^{*}}$ and Jeon-Soo Shin ${ }^{2,4}$

\section{Erratum}

In the original article [1], the following information was omitted from the funding section:

"This study was supported by a faculty research grant of Yonsei University College of Medicine for 2009 (6-2009-0101)."

\section{Author details}

'Division of Cardiology, Severance Cardiovascular Hospital, Yonsei University Health System, 50 Yonsei-ro Seodaemun-gu, Seoul 120-752, Republic of Korea. ${ }^{2}$ Departments of Microbiology, Yonsei University College of Medicine, 50 Yonsei-ro Seodaemun-gu, Seoul 120-752, Republic of Korea. ${ }^{3}$ Departments of Nuclear Medicine, Yonsei University College of Medicine, 50 Yonsei-ro Seodaemun-gu, Seoul 120-752, Republic of Korea. ${ }^{4}$ Severance Biomedical Science Institute, Yonsei University Health System, 50 Yonsei-ro

Seodaemun-gu, Seoul 120-752, Republic of Korea.

Received: 19 October 2016 Accepted: 28 October 2016

Published online: 04 November 2016

\section{Reference}

1. Yang, et al. Glycyrrhizin, inhibitor of high mobility group box-1, attenuates monocrotaline-induced pulmonary hypertension and vascular remodeling in rats. Respir Res. 2016;15(1):148.

\footnotetext{
* Correspondence: hjchang@yuhs.ac

${ }^{1}$ Division of Cardiology, Severance Cardiovascular Hospital, Yonsei University Health System, 50 Yonsei-ro Seodaemun-gu, Seoul 120-752, Republic of Korea

${ }^{4}$ Severance Biomedical Science Institute, Yonsei University Health System, 50 Yonsei-ro Seodaemun-gu, Seoul 120-752, Republic of Korea
} 